\section{Severe and delayed-onset acneiform eruptions as an adverse reaction to regorafenib}

\author{
Haruhiko Otsuka, ${ }^{1}$ Takeshi Fukumoto, ${ }^{1}$ \\ Naomi Kiyota, ${ }^{2}$ Chihiro Takemori, ${ }^{1}$ \\ Haruki Jimbo, ${ }^{1}$ Chikako Nishigori ${ }^{1}$ \\ ${ }^{1}$ Division of Dermatology, Department of \\ Internal Related, Kobe University \\ Graduate School of Medicine, Kobe; \\ ${ }^{2}$ Department of Medical Oncology and \\ Hematology, Cancer Center, Kobe \\ University Hospital, Kobe, Japan
}

\begin{abstract}
Regorafenib is an oral multikinase inhibitor targeting several tyrosine kinase receptors including BRAF and epidermal growth factor receptor (EGFR) and is approved as a third-line treatment for metastatic gastrointestinal stromal tumor (GIST). While acneiform eruptions have been observed in patients receiving other BRAF and EGFR inhibitors, the commonly reported adverse reactions to regorafenib are fatigue and palmar-plantar erythrodysesthesia. Herein, we report, to the best of our knowledge, the first case who presented with a severe acneiform eruption 24 months after beginning regorafenib for the treatment of GIST. A 61-year-old woman developed GIST with multiple liver metastases, and she was treated with imatinib and sunitinib. However, these therapies were discontinued, and regorafenib was administered. Twenty-four months after beginning regorafenib, she developed an acneiform eruption on her back Histopathologic analysis of a skin biopsy from the back revealed neutrophilic suppurative folliculitis. Therefore, she postponed regorafenib administration for 2 months and was treated with topical application of clindamycin phosphate hydrate, which was effective. Consistent with reported evidence that the presence of acneiform eruption and the efficacy of EGFR inhibitors are positively associated, regorafenib had good anticancer activity in our patient. Ultimately, we found that although regorafenib-associated skin toxicities usually appear within 1 month of treatment, patients potentially can present with delayed-onset acneiform eruptions even 24 months later.
\end{abstract}

\section{Introduction}

Regorafenib is a multikinase inhibitor that targets receptor tyrosine kinases, including BRAF and epidermal growth factor receptor (EGFR). ${ }^{1-3}$ While acneiform eruptions have been observed in patients receiving other BRAF and EGFR inhibitors, the commonly reported adverse reactions to regorafenib are fatigue and palmar-plantar erythrodysesthesia; cases of acneiform eruptions are rarely reported. ${ }^{1,4,5}$

Surgical resection is the primary indication for gastrointestinal stromal tumors (GIST). However, regorafenib has been approved as a third-line treatment for metastatic GIST. ${ }^{1,3}$ Herein, we report, to the best of our knowledge, the first case of severe and delayed acneiform eruptions after 24 months of treatment with regorafenib for GIST.

\section{Case Report}

A 61-year-old woman was diagnosed with a small intestine tumor by magnetic resonance imaging. After partial resection, the tumor was diagnosed as a high-risk GIST. The patient developed multiple liver metastases, and imatinib (400 mg/day) was administered as a first-line treatment for 23 months, but was discontinued due to tumor progression. Sunitinib (50 mg/day) was administered as a second-line treatment but was discontinued after the patient developed malaise and hand-foot syndrome. After salvage stereotactic radiation therapy targeting a single progressive metastatic lesion, imatinib (200 mg/day) was reintroduced as a third-line treatment before being discontinued due to tumor progression. Regorafenib (160 mg/day) was administered as a fourth-line treatment. One month later, the dose was reduced to $120 \mathrm{mg} /$ day due to side effects, including hand-foot syndrome. Seven months after beginning regorafenib, tumor progression required radiation therapy twice (50 Gy/4 Fr and 45 Gy/15 Fr, respectively).

Twenty-four months after beginning regorafenib, the patient developed monomorphic pink and brown papules and pustules on her back (Figure 1A-C), diagnosed as neutrophilic suppurative folliculitis (Figure 1D,E) by histopathologic analysis of a hematoxylin-eosin stained skin biopsy specimen. Grocott's methenamine silver staining and periodic-acid-Schiff staining were negative (Figure 1F,G), while Gram staining was partially positive (Figure $1 \mathrm{H})$. The patient had no history of acne on her back and reported no other likely causes
Correspondence: Takeshi Fukumoto, Division of Dermatology, Department of Internal Related, Kobe University Graduate School of Medicine, 7-5-2 Kusunoki-cho, Chuo-ku, Kobe 650-0017, Japan.

Tel.: +81.78.3826134 - Fax: +81.78.3826149. E-mail: fuku@med.kobe-u.ac.jp

Key words: Regorafenib, Acneiform Eruptions; Drug Eruptions; Adverse Drug Reaction; Skin Diseases.

Contributions: The authors contributed equally.

Conflict of interest: The authors declare no potential conflict of interest.

Funding: None.

Patient consent for publication: The patient has given informed consent for its publication.

Availability of data and material: Data and materials are available by the authors.

Please cite this article as: Otsuka $H$, Fukumoto T, Kiyota $N$, et al. Severe and delayed-onset acneiform eruptions as an adverse reaction to regorafenib. Dermatol Rep 2022;14:9303.

Received for publication: 30 June 2021. Accepted for publication: 7 July 2021.

This work is licensed under a Creative Commons Attribution-NonCommercial 4.0 International License (CC BY-NC 4.0).

(C) Copyright: the Author(s), 2022

Licensee PAGEPress, Italy

Dermatology Reports 2022; 14:9303

doi:10.4081/dr.2022.9303

Publisher's note: All claims expressed in this article are solely those of the authors and do not necessarily represent those of their affiliated organizations, or those of the publisher, the editors and the reviewers. Any product that may be evaluated in this article or claim that may be made by its manufacturer is not guaranteed or endorsed by the publisher.

for the development of acne. Therefore, we diagnosed acneiform eruptions caused by regorafenib, postponed regorafenib treatment for 2 months, and prescribed topical application of clindamycin phosphate hydrate. The acneiform eruptions significantly improved after 1 month and disappeared after 3 months.

\section{Discussion and conclusions}

Acneiform eruptions are a known adverse reaction to BRAF and EGFR 

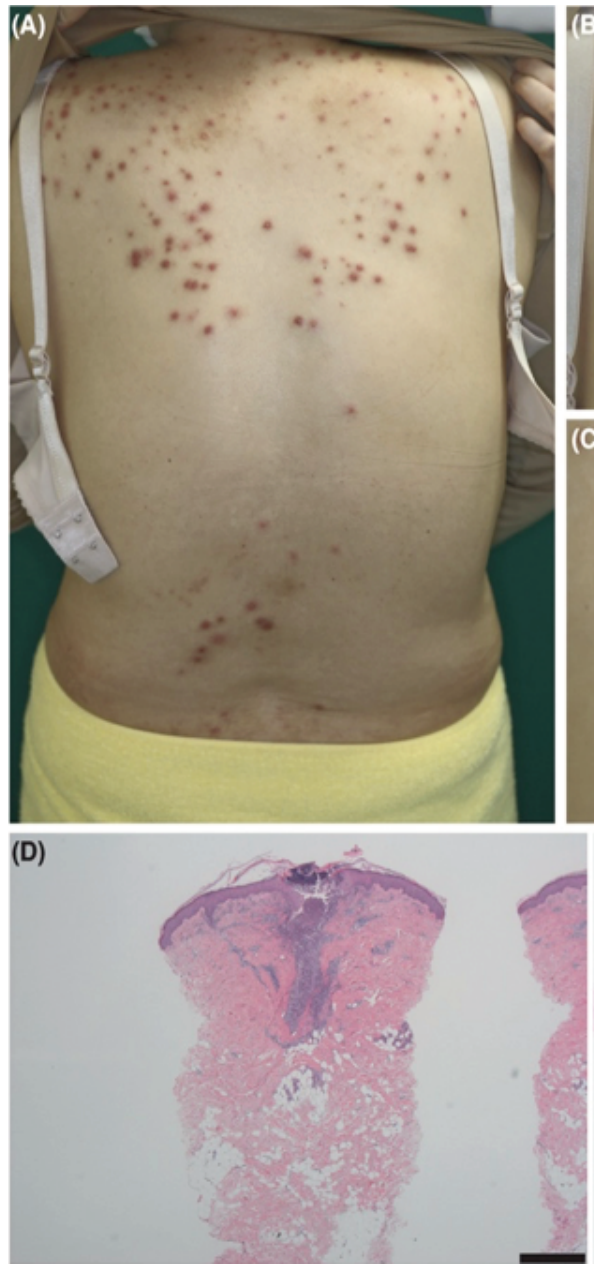
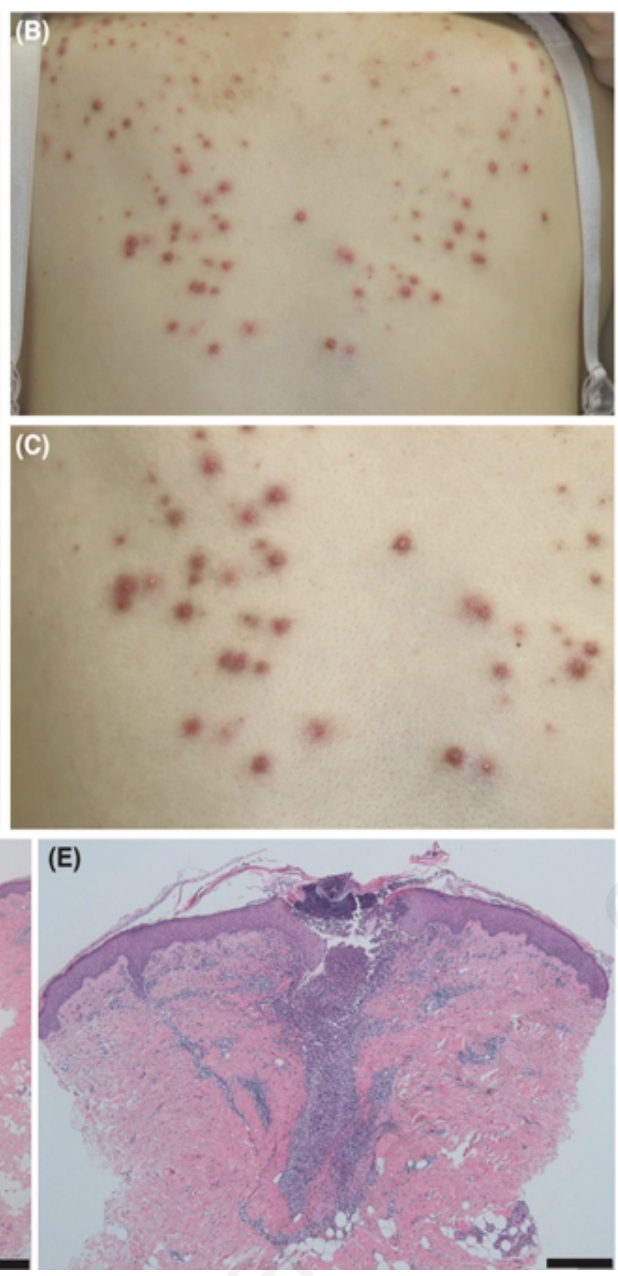

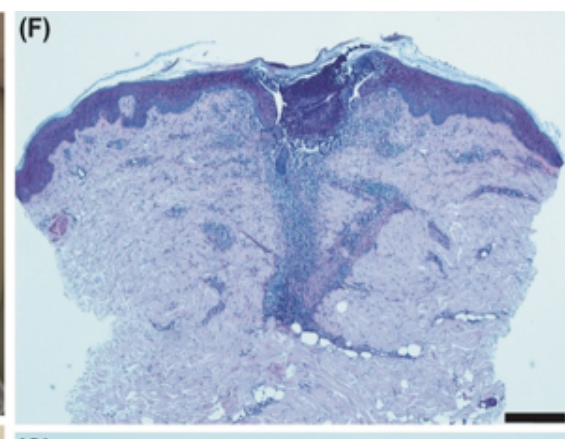

(G)
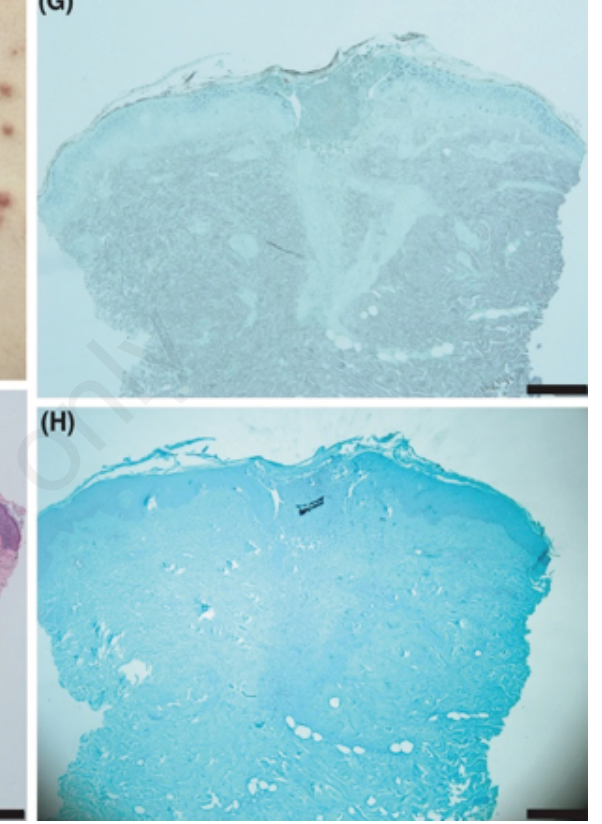

Figure 1. Clinical and histopathological features. A-C) Monomorphic pink and brown erythematous papular and pustular eruptions on the patient's back. D,E) Hematoxylin-eosin staining of a skin biopsy specimen from the patient's back revealed concentrated neutrophilic inflammation under the cornified layer, disruption of the follicular epithelium, liquefaction degeneration of the dermoepidermal junction, and infiltration of neutrophils, lymphocytes, and histiocytes around the follicle of the dermis $(\mathrm{D}: \times 20$, scale bar $=400$ $\mu \mathrm{m}, \mathrm{E}: \times 40$, scale bar $=400 \mu \mathrm{m})$. F) The results of Grocott's methenamine silver stain were negative $(\times 40$, scale bar $=400 \mu \mathrm{m})$. G) The results of periodic acid-Schiff staining were negative $(\times 40$, scale bar $=400 \mu \mathrm{m}) . \mathrm{H})$ Gram-positive bacteria were observed in the folliculitis $(\times 40$, scale bar $=400 \mu \mathrm{m})$.

inhibitors. ${ }^{2,4,5}$ BRAF inhibitors are believed to cause acneiform eruptions by paradoxical activation of the mitogen-activated protein kinase pathway via CRAF, resulting in follicular keratinocyte proliferation. ${ }^{5}$ EGFR inhibitors may cause acneiform eruptions by inhibiting EGFR on undifferentiated keratinocytes in the epidermis and on follicular keratinocytes, promoting apoptosis in keratinocytes and inducing perifollicular inflammation. ${ }^{4}$ Other studies have found a positive association between the presence of acneiform eruptions and the efficacy of the aforementioned chemotherapy agents ${ }^{4}$; our case supports this link, as regorafenib consistently exerted a strong antitumor effect in our patient.

Ultimately, we found that although regorafenib-associated skin toxicities usually appear within 1 month of treatment, ${ }^{1}$ the patients potentially can present with delayed-onset acneiform eruptions even 24 months later. Treatment with antibiotic ointment and postponed regorafenib administration improved these eruptions in our patient.

\section{References}

1. Chamberlain F, Farag S, WilliamsSharkey C, et al. Toxicity management of regorafenib in patients with gastrointestinal stromal tumour (GIST) in a tertiary cancer centre. Clin Sarcoma Res 2020;10:1.

2. Liu YC, Tsai JJ, Weng YS et al. Regorafenib suppresses epidermal growth factor receptor signaling-modulated progression of colorectal cancer.
Biomed Pharmacother 2020;128: 110319.

3. Demetri GD, Reichardt P, Kang YK et al. Efficacy and safety of regorafenib for advanced gastrointestinal stromal tumours after failure of imatinib and sunitinib (GRID): an international, multicentre, randomised, placebo-controlled, phase 3 trial. Lancet 2013;381: 295-302.

4. Brodell LA, Hepper D, Lind A et al. Histopathology of acneiform eruptions in patients treated with epidermal growth factor receptor inhibitors. J Cutan Pathol 2013;40:865-70.

5. Ansai O, Fujikawa H, Shimomura Y et al. Case of severe acneiform eruptions associated with the BRAF inhibitor vemurafenib. J Dermatol 2017;44:e15e6. 